\title{
Light Emission by Nonequilibrium Bodies: Local Kirchhoff Law
}

\author{
Jean-Jacques Greffet, ${ }^{1, *}$ Patrick Bouchon, ${ }^{2}$ Giovanni Brucoli, ${ }^{1}$ and François Marquier ${ }^{1}$ \\ ${ }^{1}$ Laboratoire Charles Fabry, Institut d'Optique Graduate School, CNRS, \\ Université Paris-Saclay, 91127 Palaiseau, France \\ ${ }^{2}$ ONERA-The French Aerospace Lab, F-91761 Palaiseau, France
}

(Received 21 December 2017; published 6 April 2018)

\begin{abstract}
The goal of this paper is to introduce a local form of Kirchhoff law to model light emission by nonequilibrium bodies. While absorption by a finite-size body is usually described using the absorption cross section, we introduce a local absorption rate per unit volume and also a local thermal emission rate per unit volume. Their equality is a local form of Kirchhoff law. We revisit the derivation of this equality and extend it to situations with subsystems in local thermodynamic equilibrium but not in equilibrium between them, such as hot electrons in a metal or electrons with different Fermi levels in the conduction band and in the valence band of a semiconductor. This form of Kirchhoff law can be used to model (i) thermal emission by nonisothermal finite-size bodies, (ii) thermal emission by bodies with carriers at different temperatures, and (iii) spontaneous emission by semiconductors under optical (photoluminescence) or electrical pumping (electroluminescence). Finally, we show that the reciprocity relation connecting light-emitting diodes and photovoltaic cells derived by Rau is a particular case of the local Kirchhoff law.
\end{abstract}

DOI: 10.1103/PhysRevX.8.021008

Subject Areas: Metamaterials, Optics, Plasmonics

\section{INTRODUCTION}

Thermal emission can be conveniently described using Kirchhoff law, which states that the emissivity is equal to the absorptivity for isothermal bodies. It has been used to analyze and design novel thermal sources with unprecedented properties. The emission spectrum can be tailored for different types of applications, such as infrared sources or sources for thermophotovoltaics [1-7]. The directivity of a source can also be controlled by taking advantage of the spatial coherence due to surface waves $[8,9]$. This has been observed experimentally for many systems based on the interplay between surface waves and gratings [10-16]. Many other schemes have been subsequently implemented using planar interfaces [17-20], guided waves and gratings [21], photonic crystals [22], and magnetic polaritons [23]. Following the pioneering work by the group of Hasman [24], the polarization of thermal radiation has been investigated [25-27]. Finally, it has become possible to modulate at high frequency the intensity emitted by thermal sources by modulating the emissivity [28]. A proof of principle has been reported in Ref. [29] and a very effective source operating up to $600 \mathrm{kHz}$ has been reported [30]. All these

*jean-jacques.greffet@institutoptique.fr

Published by the American Physical Society under the terms of the Creative Commons Attribution 3.0 License. Further distribution of this work must maintain attribution to the author(s) and the published article's title, journal citation, and DOI. developments can be analyzed using Kirchhoff law [31], which expresses the equality of absorptivity and emissivity. This law is valid provided that the emitter is in local thermodynamic equilibrium at a uniform temperature $T$ and provided that the emitting medium is reciprocal. The fate of Kirchhoff law for nonreciprocal materials has been studied recently $[32,33]$.

Despite all these applications, Kirchhoff law suffers from a number of limitations. It cannot be used to predict emission by a nonisothermal body. It cannot be used to predict thermal emission by systems where different temperatures can be defined (e.g., systems with hot electrons). It cannot be used to predict emission in the presence of quasi-Fermi levels for electrons in the conduction and valence band of a semiconductor. Its validity has also been questioned by some authors. In its original form [31], Kirchhoff law was derived using geometrical optics. The purpose of this paper is to clarify the validity conditions of Kirchhoff law and to extend it to all these nonequilibrium situations. With this aim, we use the local form of Kirchhoff law first derived by Rytov et al. [34] and extend it to the case of subsystems in local thermodynamic equilibrium.

An important issue regarding thermal emission is the case of anisothermal bodies with a temperature field that can vary in space and time $T(\mathbf{r}, t)$. Kirchhoff law cannot be used to deal with these situations. Here, we introduce a local Kirchhoff law establishing the equality between the local absorption rate and the local emission rate in any finite-size body with arbitrary shape, orientation, and structure. 
Another limitation of Kirchhoff law is the case of bodies where different subsystems (e.g., electrons, excitons, phonons) are at different temperatures at a given position. This is, for instance, the case of hot electrons in a transistor under a strong driving field or in a metallic nanosphere after the absorption of a 100-fs pulse. The local form of Kirchhoff law will be extended to provide a rigorous framework to analyze and optimize light emission by these systems.

Another interesting application of Kirchhoff law is electroluminescence by light-emitting diodes (LEDs). While the brightness and spectral properties of a thermal source and an electroluminescent source are significantly different, electroluminescence is due to the same electronhole recombination processes as thermal radiation. Once photons are emitted, their extraction from the emitting body is the same in both cases. It is known that electroluminescence (and photoluminescence) by a semiconductor slab is given by a modified Kirchhoff law including a chemical potential for the photons. More explicitly, it is given by the product of the absorptivity by $I_{b}(T, \omega-\mu / \hbar)$ [35,36], where $I_{b}$ is the blackbody radiance, $T$ is the body temperature, $\omega$ the emitting frequency, $\mu$ the (uniform) difference between quasi-Fermi levels, also called photon chemical potential, and $\hbar$ is the Planck constant. Here, we use the local form of Kirchhoff law to extend the result to inhomogeneous chemical potentials and to an arbitrary geometry of the emitter so that resonant cavities or antennas can be accounted for. Finally, we show that an important reciprocity property introduced by Rau [37] connecting light-emitting diodes and photovoltaics detectors $[38,39]$ follows naturally from the local Kirchhoff law.

The paper is organized as follows. The Sec. II presents the local form of Kirchhoff law. While the derivation can be found in Ref. [34], we provide an alternative self-contained derivation in the Appendix for the sake of completeness. In Sec. III, we explore the implications of the derivation discussing the validity conditions of Kirchhoff law, the concept of super-Planckian emission, and the extension to anisotropic media. Section IV addresses the issue of nonequilibrium systems, both in terms of nonisothermal bodies and in terms of different subsystems with different temperatures. In Sec. V, we extend Kirchhoff law to deal with electroluminescence and photoluminescence by using the concept of photon chemical potential.

\section{LOCAL FORM OF KIRCHHOFF LAW}

The aim of this section is to provide a local model of thermal emission by a body of arbitrary shape in the framework of fluctuational electrodynamics [34,40]. The key result is to express the spontaneous emission in a given mode (direction $\mathbf{u}$, frequency $\omega$, and polarization $l$ ) as an integral over the volume of the emitting body. The emission rate of each volume element will be given in terms of the power absorbed by that volume element when it is illuminated by the reciprocal mode $(-\mathbf{u}, \omega, l)$. The equality between a local emission rate and a local absorption rate is called hereafter the local form of Kirchhoff law. Let us provide some physical insight on the origin of the connection between emission, which is a thermodynamic phenomenon dependent on temperature, and absorption, which is computed in the framework of coherent electromagnetic optics. It results essentially from two reciprocity relations. Any emission process corresponds to a transition between an excited state and an unoccupied lower-energy state. The corresponding matrix element is the same for emission and absorption. This first reciprocity property shows up in the fluctuation-dissipation theorem which connects the cross-spectral density $W_{j_{n}, j_{m}}$ of the current density fluctuations and the material absorption spectrum proportional to $\operatorname{Im}[\epsilon(\mathbf{r}, \omega)]$ :

$$
W_{j_{n}, j_{m}}=2 \omega \epsilon_{0} \operatorname{Im}\left[\epsilon\left(\mathbf{r}^{\prime}, \omega\right)\right] \Theta\left[T\left(\mathbf{r}^{\prime}\right), \omega\right] \delta\left(\mathbf{r}-\mathbf{r}^{\prime}\right) \delta_{n m},
$$

where $\Theta\left[T\left(\mathbf{r}^{\prime}\right), \omega\right]=\hbar \omega /\left\{\exp \left[\hbar \omega / k_{B} T\left(\mathbf{r}^{\prime}\right)\right]-1\right\} \quad$ and $T\left(\mathbf{r}^{\prime}\right)$ is the temperature at point $\mathbf{r}^{\prime}$. This quantity is defined for positive and negative frequencies. The Fourier amplitude conventions are specified in the Appendix. Assuming the material to be local and isotropic, we get the terms $\delta\left(\mathbf{r}-\mathbf{r}^{\prime}\right) \delta_{n m}$. This fluctuation-dissipation relation is the key step connecting thermodynamic fluctuations and linear response theory. The second key reciprocity property is the reciprocity of the fields obeying Maxwell equations. This is better understood by looking at Fig. 1 .

The basic idea is that a dipole moment $\mathbf{p}_{1}$ located at $\mathbf{r}^{\prime}$ generates a field $\mathbf{E}_{1}$ at $\mathbf{r}$. Conversely, a dipole moment $\mathbf{p}_{2}$ located at $\mathbf{r}$ generates a field $\mathbf{E}_{2}$ at the position $\mathbf{r}^{\prime}$. It follows from reciprocity that these two fields satisfy $\mathbf{p}_{1} \cdot \mathbf{E}_{2}=$ $\mathbf{p}_{2} \cdot \mathbf{E}_{1}[40,41]$. In others words, by exchanging pointlike sources and detectors, the amplitudes delivered by the detectors are unchanged. By considering a source inside a body and a pointlike detector outside the body, the reciprocity theorem implies that the presence of the body modifies in the same manner emission and absorption. For instance, if we consider light emitted or absorbed by some

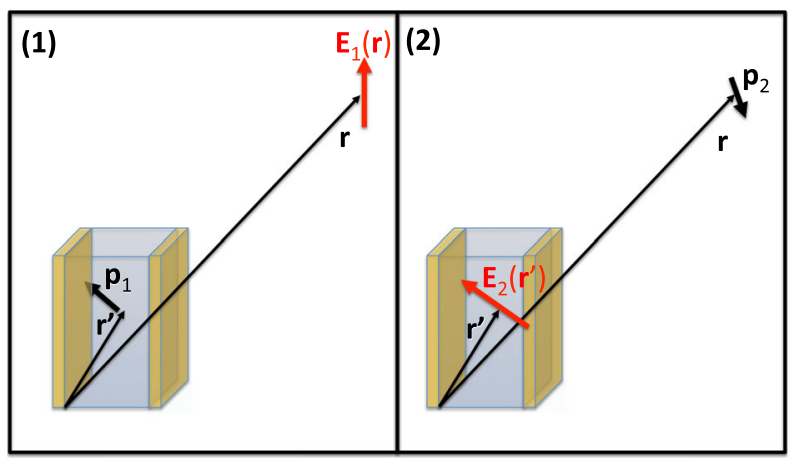

FIG. 1. Illustration of two reciprocal situations (1) and (2) obtained upon exchanging source and detector positions. The cavity is an example of an optical system made of reciprocal media. 
absorbing gas filling a Fabry-Perot cavity, Kirchhoff law will be the result of two facts: (i) the same transitions are involved in emission and absorption and (ii) the cavity satisfies reciprocity so that the cavity has the same enhancement (for molecules located at an antinode) or inhibition (for molecules located at a node) effect for absorption and emission. It is important to realize that Kirchhoff law deals with the absorptivity and emissivity of a body. It results from the interplay between microscopic properties of the material (e.g., the gas) and macroscopic properties of the body (e.g., the cavity).

When dealing with bodies much larger than the wavelength, it is possible to use locally the concept of flat interface between two homogeneous media and use locally Fresnel reflection factors. However, this geometrical approach breaks down for subwavelength bodies so that the original derivation is no longer applicable. In electromagnetic optics, the general concept to characterize the absorption of a linearly polarized monochromatic plane wave by any finite-size body with volume $V$ is the concept of absorption cross section $\sigma_{\mathrm{abs}}^{(l)}$ [42]. It connects the absorbed power $P_{\text {abs }}$ with the incident Poynting vector flux as follows:

$$
P_{\mathrm{abs}}^{(l)}(\omega)=\sigma_{\mathrm{abs}}^{(l)}\left(\mathbf{u}_{\mathrm{inc}}, \omega\right) \frac{\epsilon_{0} c\left|\mathbf{E}_{\mathrm{inc}}^{(l)}(\omega)\right|^{2}}{2},
$$

where $\mathbf{u}_{\text {inc }}$ is the incident direction (see Fig. 2), $\omega$ is the circular frequency, and the superscript $(l)$ specifies the polarization state $l=s, p$. We note that this concept does not provide any information about the position where absorption takes place in the body. An alternative form of the absorbed power is given by the integral over volume $V$ of the dissipation rate per unit volume [40]:

$$
P_{\mathrm{abs}}^{(l)}(\omega)=\int_{V} \operatorname{Im}\left[\epsilon\left(\mathbf{r}^{\prime}, \omega\right)\right] \frac{\omega \epsilon_{0}}{2}\left|\mathbf{E}^{(l)}\left(\mathbf{r}^{\prime}, \omega\right)\right|^{2} d^{3} \mathbf{r}^{\prime},
$$

where $\mathbf{E}^{(l)}\left(\mathbf{r}^{\prime}\right)$ is the field in the body illuminated by an $l$-polarized plane wave with incident field amplitude $E_{\text {inc }}^{(l)}$ and $\epsilon\left(\mathbf{r}^{\prime}, \omega\right)$ is the permittivity. This form describes

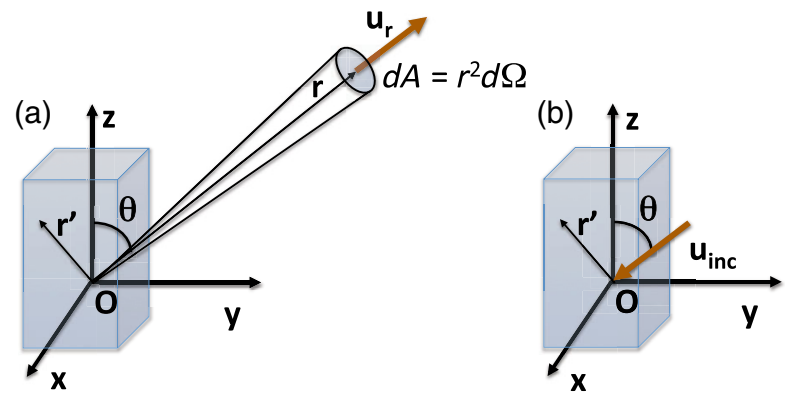

FIG. 2. Sketch of the system. A finite-size volume $V$ radiates in the solid angle $d \Omega$ subtended by the surface $d A$. explicitly where the absorption takes place but is not related explicitly to the incident field so that it cannot be expressed in terms of cross section. We now seek a connection between the incident field $\mathbf{E}_{\text {inc }}$ and the field in the absorber $\mathbf{E}\left(\mathbf{r}^{\prime}\right)$. We show in the Appendix that the existence of a linear relation allows one to cast the absorption cross section in the form:

$$
\sigma_{\mathrm{abs}}^{(l)}\left(\mathbf{u}_{\mathrm{inc}}, \omega\right)=\int_{V} d^{3} \mathbf{r}^{\prime} \alpha_{\mathrm{abs}}^{(l)}\left(\mathbf{u}_{\mathrm{inc}}, \mathbf{r}^{\prime}, \omega\right),
$$

where $\alpha_{\mathrm{abs}}^{(l)}$ appears as an absorption cross-section density so that it has the dimension of the inverse of a length. If we are interested in the absorption of a beam in a homogeneous absorbing medium, the absorption cross-section density $\alpha_{\mathrm{abs}}^{(l)}$ is nothing but the usual absorption coefficient given by $4 \pi \operatorname{Im}(n) / \lambda$, where $n$ is the refractive index. However, if we are interested in the absorption in a resonant microstructure, such a simple form cannot be used. The absorption crosssection density is no longer a material intrinsic property but depends on the body shape and orientation.

We now turn to the power emitted by the body in the solid angle $d \Omega$ (see Fig. 2). Using the fluctuational electrodynamics framework, we show in the Appendix that it can be cast in the form:

$P_{e}^{(l)}=\int_{0}^{\infty} d \omega \int_{V} \int_{4 \pi} \eta^{(l)}\left(\mathbf{u}_{r}, \mathbf{r}^{\prime}, \omega\right) \frac{I_{b}\left[T\left(\mathbf{r}^{\prime}\right), \omega\right]}{2} d^{3} \mathbf{r}^{\prime} d \Omega$,

where we have introduced the blackbody radiance $I_{b}\left[T\left(\mathbf{r}^{\prime}\right), \omega\right]=\left[\omega^{2} /\left(4 \pi^{3} c^{2}\right)\right]\left\{(\hbar \omega) /\left[\exp \left(\hbar \omega / k_{B} T\right)-1\right]\right\}$ and the local polarized emissivity density $\eta^{(l)}\left(\mathbf{u}_{r}, \mathbf{r}^{\prime}, \omega\right)$. Note that we have introduced the polarized blackbody radiance $I_{b} / 2$, which is half the blackbody radiance. Both quantities, the absorption cross-section density $\alpha^{(l)}$ and the emissivity density $\eta^{(l)}$, are polarized, directional, and monochromatic. It has been shown that these quantities are equal [34]. This result is the local form of Kirchhoff law:

$$
\eta^{(l)}\left(\mathbf{u}_{r}, \mathbf{r}^{\prime}, \omega\right)=\alpha^{(l)}\left(-\mathbf{u}_{r}, \mathbf{r}^{\prime}, \omega\right) .
$$

For the sake of completeness, we provide an alternative derivation of this result in the Appendix, where we emphasize the role of reciprocity.

\section{DISCUSSION}

\section{A. Kirchhoff law, absorption cross section, and super-Planckian emission}

The original derivation [31] by Kirchhoff is based on geometrical optics and on a budget of the energy exchanged by the body with its environment at the same temperature. It is worthwhile to point out that Helmholtz reciprocity is 
explicitly invoked by Kirchhoff, but is often omitted in subsequent derivations which then require further assumptions. A proof of Kirchhoff validity was derived in the framework of electrodynamics for a sphere with arbitrary size and material by Kattawar and Eisner [43], thereby removing the geometrical assumption for this particular case. It has also been proven without invoking geometrical optics within the scalar approximation for any planar interface separating vacuum from any complex medium satisfying reciprocity $[44,45]$. Many works have compared a direct calculation of the emission based on the fluctuational electrodynamics and a direct calculation of the absorption. So far, all these numerical calculations performed for many different systems were found to agree with Kirchhoff law [46-49]. Yet, as we discuss, there are some reports suggesting that Kirchhoff law might not be valid, so that it is important to clarify its validity domain.

Here, we use the local form of Kirchhoff law to recover the usual form. An immediate consequence of Eqs. (4)-(6) is that the total power emitted by an isothermal body in the solid angle $d \Omega$ is given by

$$
d P_{e}^{(l)}=\int_{0}^{\infty} d \omega \sigma_{\mathrm{abs}}^{(l)}\left(-\mathbf{u}_{r}, \omega\right) \frac{I_{b}(T, \omega)}{2} d \Omega .
$$

This result provides all the required information about the emission of the body and shows that it is entirely characterized by the knowledge of its absorption cross section $\sigma_{\text {abs }}^{(l)}\left(-\mathbf{u}_{r}, \omega\right)$. Hence, although Kirchhoff law was originally introduced in the framework of geometrical optics, it has been extended to any body size in the framework of fluctuational electrodynamics. We note that the definition of the absorption cross section given by Eq. (2) shows that the body can be modeled by an effective disk with area equal to $\sigma_{\text {abs }}^{(l)}\left(-\mathbf{u}_{r}, \omega\right)$ and totally absorbing (i.e., black in Kirchhoff sense). We see from Eq. (7) that the emission can also be viewed as the emission by a totally absorbing (black) body with area $\sigma_{\mathrm{abs}}^{(l)}\left(-\mathbf{u}_{r}, \omega\right)$. In summary, while geometrical optics fails for subwavelength bodies, the (geometrical optics-based) radiometry formalism can still be used provided that one uses the absorption cross section to deal with absorption and emission by the body.

There have been experimental reports and theoretical claims that thermal emission exceeding blackbody radiation in the far field is possible by using emitting materials with large density of states. Emission by a photonic crystal beyond the blackbody limit has been reported [50]. This result was refuted [51] and subsequent work [52] reported an experimental bias. More recently, it has been suggested that hyperbolic metamaterials could be used to increase thermal emission in vacuum in far field above the blackbody limit $[53,54]$. These arguments have also been refuted [55]. It should be noted that a large density of states produces a large density of energy inside the emitting body but not necessarily a larger emission. As seen in the derivation of Kirchhoff law, what matters is the coupling between the fields in the emitter and the vacuum modes outside the emitter. In order to clarify this issue, it may be useful to discuss a simple example. We consider the emission in vacuum by a lossy dielectric half-space. Assuming a refractive index $n=n^{\prime}+i n^{\prime \prime}$, the local density of electromagnetic states is given (for low losses and nondispersive media) by $n^{\prime 3} \omega^{2} /\left(\pi^{2} c^{3}\right)$. It is seen that it increases as the refractive index increases and may be much larger than in vacuum. Yet, the emission is limited to modes that are not reflected by total internal reflection. Increasing the density of the states increases the number of modes in the emitting body but does not increase the number of modes transmitted by the interface between the emitter and vacuum. In the previous example, the factor limiting the emitted power is the number of modes in vacuum, namely, in the medium where emission takes place. The power emitted by a finite-size body with area $A$ can be increased up to $n^{2} A \sigma T^{4}$ by placing the emitter in a medium of refractive index $n$. This is in full agreement with standard radiometry, as the radiance in a medium with refractive index $n$ is given by $n^{2} I_{b}(T, \omega)$. This feature can be used to extract more energy from a finite-size body by using a solid immersion lens type of geometry as discussed in Refs, [56,57].

It has been pointed out in Ref. [55] that for finite-size objects, the flux emitted could exceed the flux emitted by a blackbody with the same geometrical area. This so-called super-Planckian emission is a simple consequence of two facts well covered in textbooks [34,42]: (i) the emitted power is given by Eq. (7), (ii) the absorption cross section of a body can exceed its geometrical area. In particular, it is well known that the absorption cross section of a subwavelength sphere can be much larger than the geometrical cross section [42] due to the resonant excitation of a dipolar electric mode. For a sphere, many other modes can be resonantly excited by a plane wave leading to absorption cross sections larger than the geometrical area [6,55]. In summary, as already discussed by Bohren and Huffman [42], if the absorption is described using the absorption cross section and not the geometrical area, there is no farfield emission larger than blackbody radiation.

\section{B. Emission in a nonequilibrium situation}

In this section, we note that the original derivation of Kirchhoff law was performed by energy conservation when considering a body in equilibrium with its environment. In this section, we define the local thermodynamic equilibrium and we point out where it is used in the derivation of the emission.

The body is in local thermodynamic equilibrium if it is possible to define locally a temperature $T(\mathbf{r}, t)$ and a chemical potential $\mu(\mathbf{r}, t)$. This assumption is valid provided that the typical timescale and length scales of the variations of $T(\mathbf{r}, t)$ and $\mu(\mathbf{r}, t)$ are much larger than the thermalization 
time and scattering mean free paths, namely, that the dimensionless Knudsen number is much smaller than 1 . Under these conditions, it is possible to use the fluctuationdissipation theorem to characterize the current density.

Once the current density is known, the radiated fields can be computed in any environment provided that the optical properties are known. There is no need to have thermodynamic equilibrium between the sources and the environment.

\section{Anisotropic media}

In this section, we extend the derivation of the local Kirchhoff law to the case of anisotropic media. It is known that some anisotropic materials may display unusual optical properties associated to hyperbolic dispersion relations. It has been suggested that these features may have implications for heat transfer $[53,54]$. This point was criticized in Ref. [55]. Here, we stress that the local Kirchhoff law is valid for anisotropic media provided that the system is composed of materials with symmetric permittivity tensors $\epsilon_{n m}=\epsilon_{m n}$ as required for reciprocal media. Using the relevant form of the fluctuation-dissipation theorem,

$$
\begin{aligned}
\left\langle j_{n}(\mathbf{r}, \omega) j_{m}\left(\mathbf{r}^{\prime}, \omega^{\prime}\right)\right\rangle= & 2 \pi \delta\left(\omega+\omega^{\prime}\right) W_{j_{n}, j_{m}}\left(\mathbf{r}, \mathbf{r}^{\prime}, \omega\right), \\
W_{j_{n}, j_{m}}= & 2 \omega \epsilon_{0} \operatorname{Im}\left[\epsilon_{n m}\left(\mathbf{r}^{\prime}, \omega\right)\right] \\
& \times \delta\left(\mathbf{r}-\mathbf{r}^{\prime}\right) \Theta\left[T\left(\mathbf{r}^{\prime}\right), \omega\right],
\end{aligned}
$$

and reproducing the derivation given in the Appendix A 1, we find

$$
\begin{aligned}
\eta^{(l)}\left(\mathbf{u}_{r}, \mathbf{r}^{\prime}, \omega\right)= & \alpha^{(l)}\left(-\mathbf{u}_{r}, \mathbf{r}^{\prime}, \omega\right)=16 \pi^{2} r^{2} k \\
& \times \operatorname{Im}\left[\epsilon_{p q}\right]\left(\mathbf{r}^{\prime}, \omega\right) e_{n}^{(l)} G_{n p}\left(\mathbf{r}, \mathbf{r}^{\prime}, \omega\right) \\
& \times e_{m}^{(l)} G_{m q}^{*}\left(\mathbf{r}, \mathbf{r}^{\prime}, \omega\right) .
\end{aligned}
$$

It can be checked that the equality $\epsilon_{n m}=\epsilon_{m n}$ entails the equality $\operatorname{Im}\left[G_{n p}^{*} G_{n q} \epsilon_{p q}\right]=G_{n p}^{*} G_{n q} \operatorname{Im}\left[\epsilon_{p q}\right]$. It follows that the total emitted power is also given by the absorption cross section.

\section{EMISSION BY NONISOTHERMAL SYSTEMS}

We now consider two types of nonequilibrium bodies. We first consider systems such that a local temperature can be defined but neither isothermal nor stationary. We then consider systems where it is possible to assign different temperatures to different carriers at the same position in the body.

Emission by nonisothermal bodies can be performed by a direct calculation using the fluctuational electrodynamics framework. This type of calculation has been reported in Refs. [46-49,58-61], for instance. It can be used to deduce the temperature field from the emitted radiance by a nonisothermal system. This has been used in microwaves to recover the temperature gradient in soils or in optics to measure the temperature of shock waves in plasma. Another application consists of controlling the emitted radiance. It has been proposed [62] to emit at different wavelengths or in different directions or polarizations by heating different parts of a body. Finally, we also note that it has been shown that incandescent sources can be modulated at frequencies larger than $1 \mathrm{MHz}$ when heating objects with a size smaller than $100 \mathrm{~nm}$ surrounded by antennas designed to increase the absorption cross section [63]. The usual Kirchhoff law, which is valid for isothermal bodies, cannot be used to deal with all these applications. Instead, the local Kirchhoff law can be used to compute the power emitted by a body with any spatial and temporal temperature distribution using the absorption cross-section density:

$d P_{e}^{(l)}=\int_{0}^{\infty} d \omega \int_{V} d^{3} \mathbf{r}^{\prime} \alpha^{(l)}\left(-\mathbf{u}_{r}, \mathbf{r}^{\prime}, \omega\right) \frac{I_{b}\left[T\left(\mathbf{r}^{\prime}, t\right), \omega\right]}{2} d \Omega$.

Finally, we push the generalization of Kirchhoff law to anisothermal systems one step further by considering the case where different excitations (e.g., electrons, excitons, phonons) are at different temperatures at the same position. A typical example is a system of hot electrons in a metal or in a semiconductor. It is possible to define a temperature for the electrons and a temperature for the lattice. This is the so-called two-temperatures model. In that case, we have two different temperatures at the same point but for different subsystems. Thermal emission by hot electrons has been observed in a variety of systems, such as tunneling tips [64,65], graphene [66,67], and quantum wells [68]. These issues are of particular importance for transient regimes with different relaxation times for different carriers. It is possible to include these effects in a Kirchhoffbased model of emission if we know the contribution of the different subsystems to the absorption. Let us consider a system composed of electrons and a lattice at temperatures $T_{\mathrm{el}}$ and $T_{\mathrm{la}}$, respectively. Assuming that the imaginary part of the permittivity can be written as $\operatorname{Im}(\epsilon)=\operatorname{Im}\left(\epsilon_{\mathrm{el}}\right)+$ $\operatorname{Im}\left(\epsilon_{\mathrm{la}}\right)$, we obtain the following form of the cross-spectral density of the current $W_{j_{n}, j_{m}}$ :

$$
\begin{aligned}
\left\langle j_{n}(\mathbf{r}, \omega) j_{m}\left(\mathbf{r}^{\prime}, \omega^{\prime}\right)\right\rangle & =2 \pi \delta\left(\omega+\omega^{\prime}\right) W_{j_{n}, j_{m}}, \\
W_{j_{n}, j_{m}} & =2 \omega \epsilon_{0}\left\{\operatorname{Im}\left[\epsilon_{\mathrm{el}}\left(\mathbf{r}^{\prime}, \omega\right)\right] \Theta\left[T_{\mathrm{el}}\left(\mathbf{r}^{\prime}, t\right), \omega\right]\right. \\
& \left.+\operatorname{Im}\left[\epsilon_{\mathrm{la}}\left(\mathbf{r}^{\prime}, \omega\right)\right] \Theta\left[T_{\mathrm{la}}\left(\mathbf{r}^{\prime}, t\right), \omega\right]\right\} \delta\left(\mathbf{r}-\mathbf{r}^{\prime}\right) .
\end{aligned}
$$

This form can be used for timescales larger than the carrier thermalization time so that $T_{\mathrm{el}}$ and $T_{\mathrm{la}}$ can be defined and shorter than the electron relaxation time so that $T_{\mathrm{el}}$ and $T_{\mathrm{la}}$ are not equal. In summary, it is seen that if one can assign to each type of excitation (electrons, excitons, phonons) both a temperature and a contribution to the 
imaginary part of the permittivity, it is possible to use the generalized Kirchhoff law to account for thermal radiation by nonequilibrium systems.

\section{ELECTROLUMINESCENCE AND PHOTOLUMINESCENCE OF A SEMICONDUCTOR}

We now turn to the modeling of electroluminescence and photoluminescence using the local form of Kirchhoff law. While the usual picture associated to these processes involves the radiative recombination of an electron-hole pair, it is important to recognize that thermal emission at the same frequency is also related to the same microscopic process. The difference between thermal radiation, on one hand, and electroluminescence and photoluminescence, on the other hand, stems from the process promoting electrons to the conduction band. After thermalization, the band states are occupied according to a Fermi-Dirac distribution both in the conduction and in the valence band. Yet, each band has its own chemical potential called quasi-Fermi level to account for the modification of carrier density. This is possible because the thermalization takes place in typically 1 ps whereas radiative electron-hole recombination takes place in typically $1 \mathrm{~ns}$. This two quasi-Fermi levels situation is the typical regime of electroluminescence for light-emitting diodes. Knowing the occupation of the conduction and valence bands, it is possible to compute the recombination processes and therefore to derive the emitted power. This procedure has been outlined in detail by Wurfel [35] for a homogeneous medium. He introduced a generalized form of Kirchhoff law to model electroluminescence by a slab of semiconductor. This form has been experimentally verified [36].

In this section, we extend the local form of Kirchhoff law to nonequilibrium bodies in cases corresponding to electroluminescence and photoluminescence in semiconductors. We first introduce a model of the current density fluctuations accounting for the quasi-Fermi levels. We then derive a general form of the emitted power that can be used for any geometry including, for instance, resonant antennas or cavities. Finally, we derive a reciprocity relation connecting photovoltaic cells with LEDs.

\section{A. Current density fluctuations}

In this section, we derive the emitted power by a semiconductor from the knowledge of the fluctuating currents. Such an approach has already been used to deal with near-field heat transfer between two semiconductors $[69,70]$. The starting point is the cross-spectral density of the current density due to the electrons and holes [71] given by

$W_{j_{n}, j_{m}}\left(\mathbf{r}, \mathbf{r}^{\prime}, \omega\right)=2 \omega \epsilon_{0} \operatorname{Im}\left[\epsilon_{\mathrm{ib}}\left(\mathbf{r}^{\prime}, \omega, \mu\right)\right] \Theta[T, \omega, \mu] \delta\left(\mathbf{r}-\mathbf{r}^{\prime}\right) \delta_{n m}$, where we have introduced the photon chemical potential, which is the difference of quasi-Fermi levels $\mu=\mu_{c}-\mu_{v}$, where the subscripts $c, v$ stand for conduction band and valence band, respectively, and $\epsilon_{\mathrm{ib}}$ denotes the contribution of the interband transitions to the permittivity. Two quantities depend on the difference of the quasi-Fermi levels and on the temperature: the imaginary part of the permittivity and $\Theta$. The imaginary part of the permittivity involved in the formula is proportional to the losses and therefore depends on the population of the conduction band and valence bands. It is proportional to $\bar{n}_{v}-\bar{n}_{c}$, where $\bar{n}_{c, v}=$ $\left\{\exp \left[\left(E_{c, v}-\mu_{c, v}\right) /\left(k_{B} T\right)\right]+1\right\}^{-1}$. The Bose-Einstein term is modified as follows:

$$
\Theta\left[T\left(\mathbf{r}^{\prime}\right), \omega, \mu\left(\mathbf{r}^{\prime}\right)\right]=\frac{\hbar \omega}{\exp \left(\frac{\hbar \omega-\mu}{k_{B} T}\right)-1} .
$$

It is seen that $\mu$ plays the role of chemical potential for photons [35]. Finally, we stress that the model can be extended beyond semiconductors. The only requirement is the possibility of defining two quasi-Fermi levels for two bands. This approach is also used when dealing with dye molecules where two bands can also be defined with fast internal relaxation processes and slower radiative recombinations. We note that the temperature and the quasi-Fermi levels can be time dependent and spatially dependent, as discussed previously.

\section{B. Emission by a nonequilibrium semiconductor}

We now move to the general form of the polarized emission of a body characterized by a temperature field $T(\mathbf{r})$ and a chemical potential $\mu(\mathbf{r})$, omitting a possible time dependence for brevity:

$$
\begin{aligned}
d P_{e}^{(l)}= & d \Omega \int_{0}^{\infty} d \omega \int_{V} d^{3} \mathbf{r}^{\prime} \alpha_{\mathrm{ib}}^{(l)}\left(-\mathbf{u}, \mathbf{r}^{\prime}, \omega\right) \\
& \times \frac{\omega^{2}}{8 \pi^{3} c^{2}} \frac{\hbar \omega}{\exp \left(\frac{\hbar \omega-\mu}{k_{B} T}\right)-1},
\end{aligned}
$$

where the absorption cross section $\alpha_{\mathrm{ib}}^{(l)}\left(-\mathbf{u}, \mathbf{r}^{\prime}, \omega\right)$ accounts for the absorption by the interband transition. We note that this formula is written for a type of carriers so that the corresponding temperature, chemical potential, and permittivity is used. If needed, it is possible to sum over different carriers. The limiting cases $\mu=0$ and $\mu \gg k_{B} T$ correspond to thermal emission and electroluminescence or photoluminescence depending on the pumping mechanism. Note that this formula contains two types of information: (i) the nonequilibrium excitation of the emitting material, which is accounted for by the temperature and the photon chemical potential, and (ii) the efficiency of the coupling between plane waves and local sources mediated by the emitting body structure, which is accounted for by the local absorption cross section. 


\section{Reciprocity relation between LEDs and PV cells}

We now use this formulation to discuss an important connection between LEDs and PV cells. It has been shown by Rau [37] that there exists a reciprocity relation connecting light emitted by diodes and their quantum efficiency when they operate as PV cells. More precisely, if a device is optimized to emit light, then it is also optimized to absorb light and generate carriers [38,39]. Let us be more specific by recalling the definition of the external quantum efficiency of a PV cell. It is defined as the number of electrons collected by the cell divided by the number of incident photons with a given frequency. In other words, it is given by the product of the monochromatic absorptivity by the probability that the absorbed photon generates a collected charge. To compute this quantity, we need to compute the fraction of the absorption due to electron-hole pair generation processes. To proceed, we start from Eq. (3) and restrict the volume integral over the junction volume $V_{\text {junct }}$ and we consider only the contribution of interband transitions in the imaginary part of the semiconductor permittivity $\epsilon_{\mathrm{ib}}$ :

$d P_{\mathrm{abs}}^{(l)}=\int_{V_{\text {junct }}} d^{3} \mathbf{r}^{\prime} \frac{\epsilon_{0} \omega}{2} \operatorname{Im}\left[\epsilon_{\mathrm{ib}}\left(\mathbf{r}^{\prime}, \omega\right)\right]\left|\mathbf{E}^{(l)}\left(\mathbf{r}^{\prime}, \omega\right)\right|^{2}$.

After normalizing this quantity by the incident Poynting vector as in Eq. (A16), we find the polarized external quantum efficiency $\mathrm{EQE}^{(l)}$ :

$$
\mathrm{EQE}^{(l)}=\int_{V_{\text {junct }}} d^{3} \mathbf{r}^{\prime} \alpha_{\mathrm{ib}}^{(l)}\left(-\mathbf{u}, \mathbf{r}^{\prime}, \omega\right)
$$

Turning now to the emitted power as given by Eq. (14), we see that the integral is restricted to regions where the chemical potential is nonzero, namely, the junction. If we consider the case of a uniform chemical potential $\mu$ and a uniform temperature $T$, the emitted power can be cast in the form

$$
d P_{e}^{(l)}(\omega)=\mathrm{EQE}^{(l)} \frac{I_{b}[T, \omega-\mu / \hbar]}{2} d \Omega,
$$

which is Rau's reciprocity relation. In summary, we see that the reciprocity relation derived by Rau [37] between the $\mathrm{EQE}$ and the emitted power is a particular case of the local form of Kirchhoff law applied to the interband transitions $\eta_{\mathrm{ib}}^{(l)}\left(\mathbf{u}, \mathbf{r}^{\prime}, \omega\right)=\alpha_{\mathrm{ib}}^{(l)}\left(-\mathbf{u}, \mathbf{r}^{\prime}, \omega\right)$.

Finally, we conclude by discussing possible applications of the local Kirchhoff law. The majority of the best emitters and detectors have been developed using bulk materials. As far as detectors are concerned, using nanoantennas can be useful to funnel light in smaller detectors with reduced noise. For all these applications, it is required to optimize the external quantum efficiency. The previous analysis shows that the figure of merit to be optimized for both LED and PV cells is $\alpha_{\mathrm{ib}}^{(l)}\left(\mathbf{r}^{\prime}, \omega\right)$.

\section{CONCLUDING REMARKS}

We give a derivation of a local form of Kirchhoff law valid for any finite-size body in local thermodynamic equilibrium. In particular, the model can account for systems where different temperatures or quasi-Fermi levels can be defined for different subsystems. This approach provides a common framework to model emission processes, such as thermal emission, electroluminescence, or photoluminescence. We emphasize that the model accounts for all electromagnetic features of the emitting body such as modified density of states (e.g., photonic crystal, resonators, metamaterials) or resonances. We now discuss some possible applications.

We start with the implications for harnessing thermal radiation. It has been known for applications such as bolometers that absorption by a small volume of absorbing material can be increased using antennas [72]. Indeed, an antenna can capture more efficiently the incident power and funnel it into the absorber volume. This absorbed power in the presence of the antenna is then proportional to an effective absorption cross section denoted $\sigma_{\text {ant }}$. In addition, the antenna can be directional and frequency selective [72]. It follows from the local Kirchhoff law that if the absorption in the absorber volume is enhanced by the antenna, then its thermal emission is also enhanced. The total emitted power can be increased by the same factor $\sigma_{\text {ant }} / \sigma_{\text {abs }}$, which can be larger than 3 orders of magnitude [63]. Furthermore, the emission can be directional and frequency selective. We anticipate that a metasurface consisting of a periodic array of subwavelength hot objects connected to antennas could be optimized to behave as a blackbody antenna with unity emissivity while using only a very reduced amount of hot material.

Similarly, a small active region with quasi-Fermi levels can be inserted in nanoantennas. The local Kirchhoff law provides a model that accounts for both these effects and the nanoantenna effects. A potential application is the development of nanoantennas to reduce the electron-hole recombination time paving the way toward ultrafast modulation of LEDs [73]. Inasmuch as the thermalization time remains shorter than the electron-hole recombination time, the local Kirchhoff model can be used. As far as detectors are concerned, using nanoantennas can be useful to funnel light in smaller detectors with reduced noise. For both applications, it is required to optimize the absorption rate $\alpha_{\mathrm{ib}}$ integrated over the volume, or, in other words, the external quantum efficiency. We stress that this condition differs from the condition of the maximum Purcell factor and from the condition of maximum field enhancement by the antenna discussed in Ref. [74].

We point out that quasi-Fermi levels can also be defined for ensembles of organic molecules. Hence, the formalism 
we describe in the paper can be used to analyze molecular fluorescence. If we consider an assembly of molecules in an optical cavity at frequency $\omega_{\text {cav }}$, it is seen from Eq. (14) that the emitted power will diverge as the chemical potential $\mu$ approaches $\omega_{\text {cav }}$. This is what occurs in the Bose-Einstein condensation of photons reported in Ref. [75], so that the local Kirchhoff law appears to be an appropriate tool to model photon condensation. The effect of the cavity is included in the external quantum efficiency. The photon condensation bears some resemblance to a laser regime as (i) it has a threshold in the excitation ( $\mu$ is an increasing function of the pumping power) and (ii) all the radiation goes through the cavity mode after threshold so that there is a spectral narrowing only due to cavity filtering. Nevertheless, as opposed to a laser, the formula was derived under the assumption of local thermodynamic equilibrium so that photons are in local thermodynamic equilibrium with the medium which plays the role of a grand canonical reservoir $[35,76]$.

An important feature of the local Kirchhoff law is to account for time dynamics of hot carriers on timescales larger than the thermalization time (time needed to define a carrier temperature $T$ and a chemical potential $\mu$ ) but that can be much shorter than relaxation times (decay times of $T$ and $\mu$ ). Hence, it can be very useful to study the dynamics of hot electrons. In metals, this should be useful to analyze luminescence induced by absorption of short pulses [77]. In semiconductors, the possibility of increasing the efficiency of PV cells using hot electrons has motivated many studies of ultrafast dynamics using photoluminescence [78]. Here, the key idea is to extract hot electrons before they relax to the Fermi level.

In summary, the local Kirchhoff law is a practical tool to design and analyze a large class of light emission processes including thermal emission, photoluminescence, and electroluminescence when the emitter is in local thermodynamic equilibrium. It provides a simple model of light emission that accounts for the interplay between the electromagnetic properties of the emitter (resonances, antenna effect, etc) and the matter excitations which are included in the model through the imaginary part of the permittivity of the material and the subsystem's temperature and chemical potential.

\section{ACKNOWLEDGMENTS}

We acknowledge the support of the Agence Nationale de la Recherche through the Grant No. ANR-14-CE26-0023 and the support of the ONERA through the project SONS. This work was supported by the U.S. Department of Energy, Office of Basic Energy Sciences, Division of Materials Sciences and Engineering. It was performed, in part, at the Center for Integrated Nanotechnologies, an Office of Science User Facility operated for the U.S. Department of Energy (DOE) Office of Science. J.-J. G. acknowledges the support of Institut Universitaire de
France and the support of the chair Safran-Institut d'Optique Graduate School on Ultimate Photonics.

\section{APPENDIX: DERIVATION OF THE LOCAL KIRCHHOFF LAW}

In this appendix, we proceed to derive the local form of Kirchhoff law using the reciprocity of a Green tensor and the fluctuation-dissipation theorem. In the first part, we derive the emission cross-section rate. In the second part, we derive the absorption cross-section rate. Both are given in terms of the Green tensor and the imaginary part of the dielectric permittivity. From a practical point of view, the explicit forms of $\eta^{(l)}$ and $\alpha^{(l)}$ are rather cumbersome. In practice, it is possible to compute numerically $\alpha^{(l)}\left(\mathbf{u}, \mathbf{r}^{\prime}, \omega\right)$ and insert it in Eq. (10) to derive the emitted power.

\section{Emission}

We begin by calculating thermal emission from a local point $\mathbf{r}^{\prime}$ toward a point $\mathbf{r}$. To proceed, we consider that thermal fields are radiated by stationary random currents as discussed in Refs. [34,40]. It follows from the stationarity that the random radiated field is not square integrable so that its Fourier transform cannot be defined in the usual sense. However, it is known from Wiener-Khinchin theorem that the power spectral density is the Fourier transform of the correlation function. In what follows we use the notation of Ref. [40] for the spectral analysis of stationary random processes. This notation uses formally the Fourier transform of the fields:

$$
\begin{aligned}
\mathbf{E}(\mathbf{r}, t) & =\int_{-\infty}^{\infty} \frac{d \omega}{2 \pi} \mathbf{E}(\mathbf{r}, \omega) \exp (-i \omega t), \\
\mathbf{j}(\mathbf{r}, t) & =\int_{-\infty}^{\infty} \frac{d \omega}{2 \pi} \mathbf{j}(\mathbf{r}, \omega) \exp (-i \omega t)
\end{aligned}
$$

The correlation function of the Fourier transform of the current density components is then given by

$$
\begin{aligned}
\left\langle j_{n}(\mathbf{r}, \omega) j_{m}\left(\mathbf{r}^{\prime}, \omega^{\prime}\right)\right\rangle & =\left\langle j_{n}(\mathbf{r}, \omega) j_{m}^{*}\left(\mathbf{r}^{\prime},-\omega^{\prime}\right)\right\rangle \\
& =2 \pi \delta\left(\omega+\omega^{\prime}\right) W_{j_{n}, j_{m}}\left(\mathbf{r}, \mathbf{r}^{\prime}, \omega\right),
\end{aligned}
$$

where the brackets denote ensemble average and $W_{j_{n}, j_{m}}\left(\mathbf{r}, \mathbf{r}^{\prime}, \omega\right)$ is the cross-spectral density. It is given by the fluctuation-dissipation theorem:

$$
W_{j_{n}, j_{m}}\left(\mathbf{r}, \mathbf{r}^{\prime}, \omega\right)=2 \omega \epsilon_{0} \operatorname{Im}\left[\epsilon\left(\mathbf{r}^{\prime}, \omega\right)\right] \Theta\left[T\left(\mathbf{r}^{\prime}\right), \omega\right] \delta\left(\mathbf{r}-\mathbf{r}^{\prime}\right) \delta_{n m} .
$$

for a linear and isotropic medium with permittivity $\epsilon(\mathbf{r}, \omega)$. Let us note that this form of the fluctuation dissipation does not contain zero-point fluctuations and is symmetric for positive and negative frequencies. This is an effective form that is an appropriate and correct form for our case as discussed in Ref. [13]. The quantum theory of absorption 
prescribes the use of the normal order when computing the field correlation function. It follows that we have to compute the normally ordered correlation function of the current density that does not contain the zero-point fluctuations [13,71]. We consider a point $\mathbf{r}$ in the far field so that the emission direction is specified by the unit vector $\mathbf{u}_{r}=\mathbf{r} / r$. The electric field is transverse to the propagation direction $\mathbf{u}_{r}$ so that it can be described using only two components. Let us introduce two orthogonal unit vectors denoted $\mathbf{e}^{(s)}$ and $\mathbf{e}^{(p)}$ perpendicular to $\mathbf{u}_{r}$. The emitted power flowing through the area $d A$ is given by the flux of the Poynting vector:

$$
d P_{e}=\langle\mathbf{E}(\mathbf{r}, t) \times \mathbf{H}(\mathbf{r}, t)\rangle \cdot d A \mathbf{u}_{r} .
$$

In the far field, the electromagnetic field has a plane wave structure so that $\mathbf{H}(\mathbf{r}, \omega)=\epsilon_{0} c \mathbf{u}_{r} \times \mathbf{E}(\mathbf{r}, \omega)$. It follows that the Poynting vector through $d A$ can be cast in the form:

$$
\begin{aligned}
d P_{e}= & d A \epsilon_{0} c \int_{-\infty}^{\infty} \frac{d \omega}{2 \pi} \int_{-\infty}^{\infty} \frac{d \omega^{\prime}}{2 \pi} \exp \left[-i\left(\omega+\omega^{\prime}\right) t\right] \\
& \times\left\langle\mathbf{E}(\mathbf{r}, \omega) \cdot \mathbf{E}\left(\mathbf{r}, \omega^{\prime}\right)\right\rangle .
\end{aligned}
$$

The $m$ component of the electric field is given by

$$
E_{m}(\mathbf{r}, \omega)=i \mu_{0} \omega \int G_{m n}\left(\mathbf{r}, \mathbf{r}^{\prime}, \omega\right) j_{n}\left(\mathbf{r}^{\prime}, \omega\right) d^{3} \mathbf{r}^{\prime},
$$

where $G_{m n}$ is a component of the Green tensor and $j_{n}$ is the $n$ component of the current density. Throughout the paper, we use the Einstein notation so that there is a sum over repeated indices. The amplitude of the field along the unit vector $\mathbf{e}^{(l)}$ is given by

$E^{(l)}=\mathbf{e}^{(l)} \cdot \mathbf{E}=i \mu_{0} \omega \int e_{m}^{(l)} G_{m n}\left(\mathbf{r}, \mathbf{r}^{\prime}, \omega\right) j_{n}\left(\mathbf{r}^{\prime}, \omega\right) d^{3} \mathbf{r}^{\prime}$.

After inserting Eqs. (A1), (A3), and (A7) into Eq. (A5) and using $G_{m n}\left(\mathbf{r}, \mathbf{r}^{\prime},-\omega\right)=G_{m n}^{*}\left(\mathbf{r}, \mathbf{r}^{\prime}, \omega\right)$, we get the power emitted in $l$ polarization through $d A$ :

$$
\begin{aligned}
d P_{e}^{(l)}= & d A \int d^{3} \mathbf{r}^{\prime} \int_{-\infty}^{\infty} \frac{d \omega}{2 \pi}\left|e_{m}^{(l)} G_{m k}\left(\mathbf{r}, \mathbf{r}^{\prime}, \omega\right)\right|^{2} \\
& \times 2 k^{3} \operatorname{Im}\left[\epsilon\left(\mathbf{r}^{\prime}, \omega\right)\right] \Theta\left[T\left(\mathbf{r}^{\prime}\right), \omega\right],
\end{aligned}
$$

where $k=\omega / c$. We have just used the fact that the power spectral density of the current density fluctuation is related to the absorption spectrum given by $\operatorname{Im}\left[\epsilon\left(\mathbf{r}^{\prime}, \omega\right)\right]$. This is the first symmetry relation between intrinsic emission and absorption properties. We now cast the result in a form that can be compared to the standard radiometric approach. We restrict the integration over positive frequencies using $\operatorname{Im}\left[\epsilon\left(\mathbf{r}^{\prime}, \omega\right)\right]=-\operatorname{Im}\left[\epsilon\left(\mathbf{r}^{\prime},-\omega\right)\right]$ and we introduce the solid angle subtended by the area $d A, d \Omega=d A / r^{2}$ and the blackbody radiance $I_{b}$. The emitted power can be cast in the form

$d P_{e}^{(l)}=\int_{0}^{\infty} d \omega \int_{V} \eta^{(l)}\left(\mathbf{u}_{r}, \mathbf{r}^{\prime}, \omega\right) \frac{I_{b}\left[T\left(\mathbf{r}^{\prime}\right), \omega\right]}{2} d^{3} \mathbf{r}^{\prime} d \Omega$,

where we have defined the polarized spectral directional emissivity $\eta^{(l)}\left(\mathbf{u}_{r}, \mathbf{r}^{\prime}, \omega\right)$ :

$\eta^{(l)}\left(\mathbf{u}_{r}, \mathbf{r}^{\prime}, \omega\right)=16 \pi^{2} r^{2}\left|e_{m}^{(l)} G_{m k}\left(\mathbf{r}, \mathbf{r}^{\prime}, \omega\right)\right|^{2} k \operatorname{Im}\left[\epsilon\left(\mathbf{r}^{\prime}\right), \omega\right]$.

Note that we define the polarized emission as proportional to $\left\{I_{b}\left[T\left(\mathbf{r}^{\prime}\right), \omega\right]\right\} / 2$ so that the total emitted power is proportional to $\left(\eta^{s}+\eta^{p}\right)\left\{I_{b}\left[T\left(\mathbf{r}^{\prime}\right), \omega\right]\right\} / 2$.

\section{Absorption}

The goal of this section is to derive the absorption cross section of the body at a given frequency. To proceed, we consider an incident electric field generated by a pointlike deterministic monochromatic dipole with fixed complex amplitude oscillating with a circular frequency $\omega$. We compute the absorbed power in the body and the incident Poynting vector. Their ratio yields the absorption cross section at that particular frequency for these illumination conditions (direction and polarization). The dipole is located at $\mathbf{r}$ in the far field with an $l$-polarized dipole moment $p_{\text {inc }} \mathbf{e}^{(l)}$. The $m$ component of the field $\mathbf{E}^{(l)}\left(\mathbf{r}^{\prime}\right)$ generated at $\mathbf{r}^{\prime}$ is then given by

$$
E_{m}^{(l)}\left(\mathbf{r}^{\prime}\right)=G_{m n}\left(\mathbf{r}^{\prime}, \mathbf{r}, \omega\right) e_{n}^{(l)} p_{\text {inc }} \mu_{0} \omega^{2},
$$

and the amplitude of the incident field propagating in vacuum produced by the $l$-polarized electric dipole at point $\mathbf{r}$ is given by

$$
E_{\text {inc }}^{(l)}=\frac{\exp (i k r)}{4 \pi r} \mu_{0} \omega^{2} p_{\text {inc }} .
$$

It follows that

$$
E_{m}^{(l)}\left(\mathbf{r}^{\prime}\right)=G_{m n}\left(\mathbf{r}^{\prime}, \mathbf{r}, \omega\right) e_{n}^{(l)} 4 \pi r E_{\mathrm{inc}}^{(l)} \exp (-i k r) .
$$

We now use the reciprocity theorem,

$$
G_{m n}\left(\mathbf{r}^{\prime}, \mathbf{r}, \omega\right)=G_{n m}\left(\mathbf{r}, \mathbf{r}^{\prime}, \omega\right),
$$

which is the second key ingredient in the derivation. We stress that this equality is valid in the presence of any resonator, cavity, antenna, etc. provided that all the materials have symmetric permittivity tensors. The absorbed power is given by Eq. (3): 
$P_{\mathrm{abs}}^{(l)}=\int_{V} \operatorname{Im}\left[\epsilon\left(\mathbf{r}^{\prime}, \omega\right)\right] \frac{\omega \epsilon_{0}}{2}\left|e_{n}^{(l)} G_{n m}\left(\mathbf{r}, \mathbf{r}^{\prime}, \omega\right) 4 \pi r E_{\mathrm{inc}}\right|^{2} d^{3} \mathbf{r}^{\prime}$.

This result can be cast in the form

$$
P_{\mathrm{abs}}^{(l)}=\frac{\epsilon_{0} c}{2}\left|E_{\mathrm{inc}}\right|^{2} \int_{V} d^{3} \mathbf{r}^{\prime} \alpha^{(l)}\left(\mathbf{u}_{\mathrm{inc}}, \mathbf{r}^{\prime}, \omega\right)
$$

where we have defined a polarized directional absorption cross-section density $\alpha^{(l)}\left(\mathbf{u}_{\text {inc }}, \mathbf{r}^{\prime}, \omega\right)$ :

$\alpha^{(l)}\left(\mathbf{u}, \mathbf{r}^{\prime}, \omega\right)=16 \pi^{2} r^{2}\left|e_{m}^{(l)} G_{m k}\left(\mathbf{r}, \mathbf{r}^{\prime}, \omega\right)\right|^{2} k \operatorname{Im}\left[\epsilon\left(\mathbf{r}^{\prime}\right), \omega\right]$.

The incident direction is characterized by $\mathbf{u}_{\text {inc }}=$ $-\mathbf{r} / r=-\mathbf{u}_{r}$. Upon inspection, we see that

$$
\eta^{(l)}\left(\mathbf{u}_{r}, \mathbf{r}^{\prime}, \omega\right)=\alpha^{(l)}\left(-\mathbf{u}_{r}, \mathbf{r}^{\prime}, \omega\right),
$$

which is the local form of Kirchhoff law.

[1] P. J. Hesketh, J. N. Zemel, and B. Gebhart, Organ Pipe Radiant Modes of Periodic Micromachined Silicon Surfaces, Nature (London) 324, 549 (1986).

[2] M. U. Pralle, N. Moelders, M. P. McNeal, I. Puscasu, A. C. Greenwald, J. T. Daly, E. A. Johnson, T. George, D. S. Cho, I. El-Kady, and R. Biswas, Photonic Crystal Enhanced Narrow-Band Infrared Emitters, Appl. Phys. Lett. 81, 4685 (2002).

[3] I. Puscasu and W. L. Schaich, Narrow-Band, Tunable Infrared Emission from Arrays of Microstrip Patches, Appl. Phys. Lett. 92, 233102 (2008).

[4] X. Liu, T. Tyler, T. Starr, A. F. Starr, N. M. Jokerst, and W. J. Padilla, Taming the Blackbody with Infrared Metamaterials as Selective Thermal Emitters, Phys. Rev. Lett. 107, 045901 (2011).

[5] E. Rephaeli and S. Fan, Absorber and Emitter for Solar Thermophotovoltaic Systems to Achieve Efficiency Exceeding the Schockley-Queisser Limit, Opt. Express 17, 15145 (2009).

[6] A. Lenert, D. M. Bierman, Y. Nam, W. R. Chan, I. Celanovic, M. Soljacic, and E. N. Wang, A Nanophotonic Solar TherMophotovoltaic Device, Nat. Nanotechnol. 9, 126 (2014).

[7] D. Costantini, A. Lefebvre, A. L. Coutrot, I. MoldovanDoyen, J. P. Hugonin, S. Boutami, F. Marquier, H. Benisty, and J. J. Greffet, Plasmonic Metasurface for Directional and Frequency-Selective Thermal Emission, Phys. Rev. Applied 4, 014023 (2015).

[8] J. Le Gall, M. Olivier, and J.-J. Greffet, Experimental and Theoretical Study of Reflection and Coherent Thermal Emission by a SiC Grating Supporting a Surface Phonon Polariton, Phys. Rev. B 55, 10105 (1997).
[9] R. Carminati and J.-J. Greffet, Near-Field Effects in Spatial Coherence of Thermal Sources, Phys. Rev. Lett. 82, 1660 (1999).

[10] M. Kreiter, J. Oster, R. Sambles, S. Herminghaus, S. MittlerNeher, and W. Knoll, Thermally Induced Emission of Light from a Metallic Diffraction Grating, Mediated by Surface Plasmons, Opt. Commun. 168, 117 (1999).

[11] J. J. Greffet, R. Carminati, K. Joulain, J. P. Mulet, S. Mainguy, and Y. Chen, Coherent Emission of Light by Thermal Sources, Nature (London) 416, 61 (2002).

[12] F. Marquier, K. Joulain, J. P. Mulet, R. Carminati, J. J. Greffet, and Y. Chen, Coherent Spontaneous Emission of Light by Thermal Sources, Phys. Rev. B 69, 155412 (2004).

[13] K. Joulain, J. P. Mulet, F. Marquier, R. Carminati, and J. J. Greffet, Surface Electromagnetic Waves Thermally Excited: Radiative Heat Transfer, Coherence Properties and Casimir Forces Revisited in the Near Field, Surf. Sci. Rep. 57, 59 (2005).

[14] N. Dahan, A. Niv, G. Biener, Y. Gorodetski, V. Kleiner, and E. Hasman, Enhanced Coherency of Thermal Emission: Beyond the Limitation Imposed by Delocalized Surface Waves, Phys. Rev. B 76, 045427 (2007).

[15] G. Biener, N. Dahan, A. Niv, V. Kleiner, and E. Hasman, Highly Coherent Thermal Emission Obtained by Plasmonic Bandgap Structures, Appl. Phys. Lett. 92, 081913 (2008).

[16] C. Arnold, F. Marquier, M. Garin, F. Pardo, S. Collin, N. Bardou, J. L. Pelouard, and J. J. Greffet, Coherent Thermal Infrared Emission by Two-Dimensional Silicon Carbide Gratings, Phys. Rev. B 86, 035316 (2012).

[17] L. Hu, A. Schmidt, A. Narayanaswamy, and G. Chen, Effects of Periodic Structures on the Coherence Properties of Blackbody Radiation, J. Heat Transfer 126, 786 (2004).

[18] B. J. Lee, C. J. Fu, and Z. Zhang, Coherent Thermal Emission from One-Dimensional Photonic Crystals, Appl. Phys. Lett. 87, 071904 (2005).

[19] S. Enoch, J. J. Simon, L. Escoubas, Z. Elalmy, F. Lemarquis, P. Torchio, and G. Albrand, Simple Layer-by-Layer Photonic Crystal for the Control of Thermal Emission, Appl. Phys. Lett. 86, 261101 (2005).

[20] J. Drevillon and P. Ben-Abdallah, Ab Initio Design of Coherent Thermal Sources, J. Appl. Phys. 102, 114305 (2007).

[21] K. Joulain and A. Loizeau, Coherent Thermal Emission by Microstructured Waveguides, J. Quant. Spectrosc. Radiat. Transf. 104, 208 (2007).

[22] M. Laroche, R. Carminati, and J. J. Greffet, Coherent Thermal Antenna Using a Photonic Crystal Slab, Phys. Rev. Lett. 96, 123903 (2006).

[23] B. J. Lee, L. P. Wang, and Z. M. Zhang, Coherent Thermal Emission by Excitation of Magnetic Polaritons between Periodic Strips and a Metallic Film, Opt. Express 16, 11328 (2008).

[24] N. Dahan, A. Niv, G. Biener, V. Kleiner, and E. Hasman, Space-Variant Polarization Manipulation of a Thermal Emission by a $\mathrm{SiO}_{2}$ Subwavelength Grating Supporting Surface Phonon-Polaritons, Appl. Phys. Lett. 86, 191102 (2005).

[25] N. Dahan, Y. Gorodetski, K. Frischwasser, V. Kleiner, and E. Hasman, Geometric Doppler Effect: Spin-Split 
Dispersion of Thermal Radiation, Phys. Rev. Lett. 105, 136402 (2010).

[26] F. Marquier, C. Arnold, M. Laroche, J. J. Greffet, and Y. Chen, Degree of Polarization of Thermal Light Emitted by Gratings Supporting Surface Waves, Opt. Express 16, 5305 (2008).

[27] M. Makhsiyan, P. Bouchon, J. Jaeck, J. L. Pelouard, and R. Haidar, Shaping the Spatial and Spectral Emissivity at the Diffraction Limit, Appl. Phys. Lett. 107, 251103 (2015).

[28] J.-J. Greffet, Controlled Incandescence, Nature (London) 478, 191 (2011).

[29] S. Vassant, I. Moldovan-Ledoyen, F. Marquier, F. Pardo, U. Gennser, A. Cavanna, J. L. Pelouard, and J. J. Greffet, Electrical Modulation of Emissivity, Appl. Phys. Lett. 102, 081125 (2013).

[30] T. Inoue, M. De Zoysa, T. Asano, and S. Noda, Realization of Dynamic Thermal Emission Control, Nat. Mater. 13, 928 (2014).

[31] G. Kirchhoff, On the Relation between the Radiating and Absorbing Powers of Different Bodies for Light and Heat, Philos. Mag. Ser. 5 20, 1 (1860).

[32] L. Zhu and S. Fan, Near-Complete Violation of Detailed Balance in Thermal Radiation, Phys. Rev. B 90, 220301 (2014).

[33] D. A. B. Miller, L. Zhu, and S. Fan, Universal Modal Radiation Laws for All Thermal Emitters, Proc. Natl. Acad. Sci. U.S.A. 114, 4336 (2017).

[34] S. M. Rytov, Y. A. Kravtsov, and V. I. Tatarskii, Principles of Statistical Radiophysics (Springer-Verlag, Berlin, 1989), Vol. 3.

[35] P. Würfel, The Chemical Potential of Radiation, J. Phys. C 15, 3967 (1982).

[36] L. Ferraioli, P. Maddalena, E. Massera, A. Paretta, M. A. Green, A. Wang, and J. Zhao, Evidence for Generalized Kirchhoff's Law from Angle-Resolved Electroluminescence of High Efficiency Silicon Solar Cells, Appl. Phys. Lett. 85, 2484 (2004).

[37] U. Rau, Reciprocity Relation between Photovoltaic Quantum Efficiency and Electroluminescent Emission of Solar Cells, Phys. Rev. B 76, 085303 (2007).

[38] O. D. Miller, E. Yablonovitch, and S. R. Kurtz, Strong Internal and External Luminescence as Solar Cells Approach the Shockley-Queisser Limit, IEEE J. Photovoltaics 2, 303 (2012).

[39] M. A. Green, Radiative Efficiency of State of the Art Photovoltaic Cells, Prog. Photovoltaics 20, 472 (2012).

[40] L. D. Landau and E. M. Lifshitz, Statistical Physics (Pergamon Press, Oxford, 1969), Vol. 5.

[41] J. J. Greffet and R. Carminati, Image Formation in NearField Optics, Prog. Surf. Sci. 56, 133 (1997).

[42] C. F. Bohren and D. R. Huffman, Absorption and Scattering of Light by Small Particles (Wiley, New York, 1983).

[43] G. W. Kattawar and M. Eisner, Radiation from a Homogeneous Isothermal Sphere, Appl. Opt. 9, 2685 (1970).

[44] J. J. Greffet and M. Nieto-Vesperinas, Field Theory for Generalized Bidirectional Reflectivity: Derivation of Helmholtz's Reciprocity Principle and Kirchhoff's Law, J. Opt. Soc. Am. A 15, 2735 (1998).
[45] W. C. Snyder, Z. Wan, and X. Li, Thermodynamic Constraints on Reflectance Reciprocity and Kirchhoff's Law, Appl. Opt. 37, 3464 (1998).

[46] C. Luo, A. Narayanaswamy, G. Chen, and J. D. Joannopoulos, Thermal Radiation from Photonic Crystals: A Direct Calculation, Phys. Rev. Lett. 93, 213905 (2004).

[47] D. L. C. Chan, M. Soljacic, and J. D. Joannopoulos, Direct Calculation of Thermal Emission for Three-Dimensionally Periodic Photonic Crystals Slabs, Phys. Rev. E 74, 036615 (2006).

[48] L. P. Wang, S. Basu, and Z. M. Zhang, Direct and Indirect Methods for Calculating Thermal Emission from Layered Structures with Nonuniform Temperatures, J. Heat Transfer 133, 072701 (2011).

[49] S. Edalatpour and M. Francoeur, Size Effect on the Emissivity of Thin Films, J. Quant. Spectrosc. Radiat. Transf. 118, 75 (2013).

[50] S.-Y. Lin, J. Moreno, and J. G. Fleming, Three-Dimensional Photonic-Crystal Emitter for Thermal Photovoltaic Power Generation, Appl. Phys. Lett. 83, 380 (2003).

[51] T. Trupke, P. Wurfel, and M. A. Green, Comment on: ThreeDimensional Photonic-Crystal Emitter for Thermal Photovoltaic Power Generation, Appl. Phys. Lett. 84, 1997 (2004).

[52] J. G. Fleming, Addendum: Three-Dimensional PhotonicCrystal Emitter for Thermal Photovoltaic Power Generation, Appl. Phys. Lett. 86, 249902 (2005).

[53] I. S. Nefedov and L. A. Melnikov, Super-Planckian FarField Thermal Emission from Asymmetric Hyperbolic Metamaterials, Appl. Phys. Lett. 105, 161902 (2014).

[54] S. I. Maslovski, C. R. Simovski, and S. A. Tretyakov, Overcoming Blackbody Radiation Limit in Free Space: Metamaterial Superemitter, New J. Phys. 18, 013034 (2016).

[55] S.-A. Biehs and P. Ben-Abdallah, Revisiting SuperPlanckian Thermal Emission in the Far-Field Regime, Phys. Rev. B 93, 165405 (2016).

[56] N. J. Harrick and A. F. Turner, A Thin Film Optical Cavity to Induce Absorption or Thermal Emission, Appl. Opt. 9, 2111 (1970).

[57] Z. Yu, N. P. Sergeant, T. Skauli, G. Zhang, H. Wang, and S. Fan, Enhancing Far-Field Thermal Emission with Thermal Extraction, Nat. Commun. 4, 1730 (2013).

[58] S. E. Han, Theory of Thermal Emission from Periodic Structures, Phys. Rev. B 80, 155108 (2009).

[59] L. Tsang, E. Njoku, and J. A. Kong, Microwave Thermal Emission from a Stratified Medium with Nonuniform Temperature Distribution, J. Appl. Phys. 46, 5127 (1975).

[60] F. Bardati and D. Solimini, On the Emissivity of Layered Materials, IEEE Trans. Geosci. Remote Sens. GE-22, 374 (1984).

[61] K. P. Gaikovich, A. N. Reznik, V.L. Vaks, and N. V. Yurasova, New Effect in Near-Field Thermal Emission, Phys. Rev. Lett. 88, 104302 (2002).

[62] S. E. Han and D. J. Norris, Control of Thermal Emission by Selective Heating of Periodic Structures, Phys. Rev. Lett. 104, 043901 (2010).

[63] E. Sakat, L. Wojszvzyk, J. P. Hugonin, M. Besbes, C. Sauvan, and J. J. Greffet, Enhancing Thermal Radiation with Nanoantennas to Create Infrared Sources with High Modulation Rates, Optica 5, 175 (2018). 
[64] A. Downes, P. Dumas, and M. E. Welland, Measurement of High Electron Temperatures in Single Atom Metal Point Contacts by Light Emission, Appl. Phys. Lett. 81, 1252 (2002).

[65] M. Buret, A. V. Uskov, J. Dellinger, N. Cazier, M.-M. Mennemanteuil, J. Berthelot, I. V. Smetanin, I. E. Protsenko, G. Colas-des-Francs, and A. Bouhelier, Spontaneous Hot-Electron Light Emission from Electron-Fed Optical Antennas, Nano Lett. 15, 5811 (2015).

[66] M. Freitag, H.-Y. Chiu, M. Steiner, V. Perebeinos, and P. Avouris, Thermal Infrared Emission from Biased Graphene, Nat. Nanotechnol. 5, 497 (2010).

[67] Y.D. Kim et al., Bright Visible Light Emission from Graphene, Nat. Nanotechnol. 10, 676 (2015).

[68] T. Laurent, Y. Todorov, A. Vasanelli, A. Delteil, C. Sirtori, I. Sagnes, and G. Beaudoin, Superradiant Emission from a Collective Excitation in a Semiconductor, Phys. Rev. Lett. 115, 187402 (2015).

[69] K. Chen, P. Santhanam, S. Sandhu, L. Zhu, and S. Fan, Heat Flux Control and Solid State Cooling by Regulating Chemical Potential of Photons in Near-Field Electromagnetic Heat Transfer, Phys. Rev. B 91, 134301 (2015).

[70] J. DeSutter, R. Vaillon, and M. Francoeur, External Luminescence and Photon Recycling in Near-Field Thermophotovoltaics, Phys. Rev. Applied 8, 014030 (2017).

[71] C. H. Henry and R. F. Kazarinov, Quantum Noise in Photonics, Rev. Mod. Phys. 68, 801 (1996).
[72] J. Alda, J. M. Rico-Garcia, J. M. Lopez-Alonso, and G. Boreman, Optical Antennas for Nano-Photonic Applications, Nanotechnology 16, S230 (2005).

[73] K. L. Tsakmakidis, R. W. Boyd, E. Yablonovitch, and X. Zhang, Large Spontaneous Emission Enhancements in Metallic Nanostructures: Towards LEDs Faster than Lasers, Opt. Express 24, 17916 (2016).

[74] T. J. Seok, A. Jamshidi, M. Kim, S. Dhuey, A. Lakhani, H. Choo, P. J. Schick, S. Cabrini, A. M. Schwartzberg, J. Bokor, E. Yablonovitch, and M. C. Wu, Radiation Engineering of Optical Antennas for Maximum Field Enhancement, Nano Lett. 11, 2606 (2011).

[75] J. Klaers, J. Schmitt, F. Vewinger, and M. Weitz, BoseEinstein Condensation of Photons in an Optical Microcavity, Nature (London) 468, 545 (2010).

[76] J. Schmitt, T. Damm, D. Dung, F. Vewinger, J. Klaers, and M. Weitz, Observation of Grand-Canonical Number Statistics in a Photon Bose-Einstein Condensate, Phys. Rev. Lett. 112, 030401 (2014).

[77] T. Haug, P. Klemm, S. Bange, and J. M. Lupton, HotElectron Intraband Luminescence from Single Hots Spots in Noble-Metal Nanoparticle Films, Phys. Rev. Lett. 115, 067403 (2015).

[78] F. Gibelli, L. Lombez, and J.-F. Guillemoles, Accurate Radiation Temperature and Chemical Potential from Quantitative Photoluminescence Analysis of Hot Carrier Populations, J. Phys. Condens. Matter 29, 06LT02 (2017). 